\title{
ON SOME OVERVIEWS OF HETEROJUNCTION BIPOLAR TRANSISTOR TECHNOLOGY: A STUDY
}

\author{
Arpita Santra(Barman), Arnima Das, Abhijit Ghosh \\ Department of ECE \\ Narula Institute of Technology, \\ Kolkata, West Bengal, India
}

\begin{abstract}
Heterojunction Bipolar Transistor (HBT)[1] technology has become a serious player in wireless communication, power electronic equipment, mixer, and frequency synthesizer applications. HBTs extend the benefits of semiconductor bipolar transistors to considerably higher frequencies. Since the middle -1980 s, HBT technology development has targeted on reducing value and rising dependability that successively, led to numerous commercial products, such as prescalers, gate arrays, digital-to-analog converters, mux/demux chip sets, logarithmic amplifiers, RF chip sets for CDMA wireless communication systems, and power amplifiers for cellular communications. They have become a natural alternative for terribly high frequency military applications requiring a high current drive, high transconductance, high voltage handling capability, low noise oscillator, and uniform threshold voltage. Emerging HBT technologies enable the combination of an outsized amount of high performance RF circuits and high speed digital circuits on one chip. In these research paper efforts has been given to obtain better results with a novel heterojunction.
\end{abstract}

Keywords- Heterojunction, transconductance, gain, semiconductor

\section{INTRODUCTION}

Heterostructures could also be outlined as heterogeneous semiconductor structures designed from additional completely different semiconductors, in such some way that the transition region or interface between the various materials play an essential role in any device action.

The heterojunction bipolar transistor (HBT) is associate degree improvement of the bipolar Junction Transisto (BJT) that may handle signals of terribly high frequencies up to many hundred gigahertz. It is common in fashionable immoderate quick circuits, largely radio-frequency (RF) systems.

The principal difference between the BJT and HBT is the use of differing semiconductor materials for the emitter and base regions, creating a heterojunction. The result is to limit the injection of holes into the bottom region, since the potential barrier within the valence band is thus massive. Unlike BJT technology, this allows high doping to be used in the base, creating higher electron mobility while maintaining gain. The potency of the device is measured by the Kroemer [2,3] issue, after Herbert Kroemer who received a Nobel Prize for his work in this field in 2000.

Materials used for the substrate include silicon-germanium alloys and gallium arsenide, while aluminium gallium arsenide, indium phosphide and indium gallium phosphide are used for the epitaxial layers. Wide-band-gap semiconductors are especially promising, e.g. gallium nitride and indium gallium nitride.

In $\mathrm{SiGe}[4,5]$ graded heterostructure transistors, the amount of germanium in the base is graded, making the band-gap narrower at the collector than at the emitter. That tapering of the band-gap results in a field-assisted transport within the base, that speeds transport through the bottom and will increase frequency response.

A higher operating speed can open up new application areas at very high frequencies, or can be traded in for lower power dissipation, or can help to reduce the impact of process, voltage and temperature variations at lower frequencies for higher circuit dependability. SiGe HBTs area unit key devices for high-frequency low-power applications[6-8]. Compared to III-V compound semiconductor devices, they enable high density[9] and low-cost integration making them suitable for consumer applications.

Over the past few years SiGe heterojunction bipolar transistors have set out of the laboratory and gone into production in BiCMOS[10-11] processes round the world. Because of difficulties in growing lattice-matched $\mathrm{SiGe}$ alloy on $\mathrm{Si}$, this 
concept is reduced to practical reality only in the last 15 years. SiGe HBT technology was originally developed at IBM for the high-end computing market, that effort, however, failed to CMOS, primarily because of its high power consumption.

In the early Nineteen Nineties, IBM refocused its $\mathrm{SiGe}$ program towards the quickly developing communications market. Interestingly, for RF communications circuits, SiGe HBT consumes a lot of less power than CMOS to attain constant level of performance. Since then, significant progress has been made. SiGe technology is being developed and applied round the world, and is within the product roadmap of each major telecommunication company. Applications range from wired and wireless communications circuits, to disk storages, to high speed high bandwidth instrumentation. The use of distinct SiGe HBTs and amplifiers in wireless devices is common place. Integrated SiGe chips will be found in GSM and CDMA wireless handsets and base stations, wireless computer network chipsets, and high-speed 10-40 Gb/s synchronous optical network (SONET) transceivers.

To date, all SiGe HBTs are created with a vertical design within which the electrode is placed on top of the bottom and also the base on top of the collector. Although this approach has given the on top of spectacular performance, it's variety of disadvantages. These disadvantages arise from the requirement to form a contact to the collector, which is buried below the surface. A heavily doped buried layer is required at a lower place the collector to cut back the collector resistance, and epitaxy has to be used to create the lower doped collector on top of the buried layer. Epitaxy may be a terribly pricy method, and even with a very heavily doped buried layer, it is not possible to achieve very low values of collector resistance.

Table -1 Comparison of CMOS with conventional and SiGe BJTs (After Harame)

\begin{tabular}{|c|c|c|c|}
\hline Parameter & CMOS & Si BJT & SiGe BJT \\
\hline Linearity & Best & Good & Better \\
\hline Broadband noise & Poor & Good & Good \\
\hline Early voltage & Poor & OK & Good \\
\hline Tranconductance & Poor & Good & Good \\
\hline
\end{tabular}

In principle, this drawback may well be resolved by employing a lateral design within which the electrode, base and collector were placed facet by facet at the surface. Contact to the collector may then be created directly from the surface, giving a really low price of collector resistance and eliminating the requirement for collector growing. This arrangement would even be additional compatible with CMOS, wherever the MOS transistors area unit made-up employing a lateral design[12]. Silicon lateral bipolar transistors are on the market for a few time, but they tend to be low frequency devices because of the parasitic capacitances associated with them. Lateral SiGe HBTs have never been reported. To produce a high frequency lateral SiGe HBT, it is necessary to minimize parasitic capacitance and at the same time find a method of producing a lateral SiGe layer. Silicon on material (SOI) technology offers one methodology of reducing parasitic capacitance, and has delivered an extremely impressive frequency of $67 \mathrm{GHz}$ on lateral silicon bipolar transistors. Simulations of lateral SiGe HBTs on SOI substrates indicate that the lateral SiGe HBT on SOI outperforms the vertical HBT, especially in terms of speed. Confined lateral selective epitaxial growth (CLSEG) and germanium implantation are two possible methods of creating a lateral SiGe layer. Both techniques have shown promising results.

SiGe HBTs may be a hot topic inside the electronics community owing to its applications potential inside integrated circuits in operation at radio frequencies. Applications vary from high speed optical networking to wireless communication devices.

The addition of chemical element to semiconductor technologies to create semiconductor chemical element ( $\mathrm{SiGe}$ ) devices has created a revolution within the semiconductor trade. These transistors form the enabling devices in a wide range of products for wireless and wired communications.

Silicon-Germanium ( $\mathrm{SiGe}$ ) technology is that the propulsion behind the explosion in affordable, lightweight, personal communications devices like digital wireless handsets, as well as other entertainment and information technologies like digital set-top boxes, Direct Broadcast Satellite (DBS), automobile collision avoidance systems, and personal digital assistants. SiGe extends the life of wireless phone batteries, and allows smaller and more durable communication devices. A product combining the capabilities of cellular phones, global positioning, and Internet access in one package, is being designed using SiGe technology. These multifunction, low-cost, mobile shopper devices capable of act over voice and knowledge networks represent a key part of the longer term of computing.

Methodology: In this work $\mathrm{SiGe}$ and Germanium has been considered as the base material of the Heterojunction design. Very low amount of doping of Ge into Si gives very good result. Also studies has been made to see if the lattice matching of this two semiconductor material is possible or not. MATLAB has been utilized to obtain the results.

\section{RESUlTS}

In the analysis stage studies has been made to obtain the gain variation with the percentage of doping into the primary semiconductor( $\mathrm{Si})$. In the figure 1 it is very clear that gain is high and with the variation of doping levels by $5 \%$ to $15 \%$ of doping on primary semiconductor. 


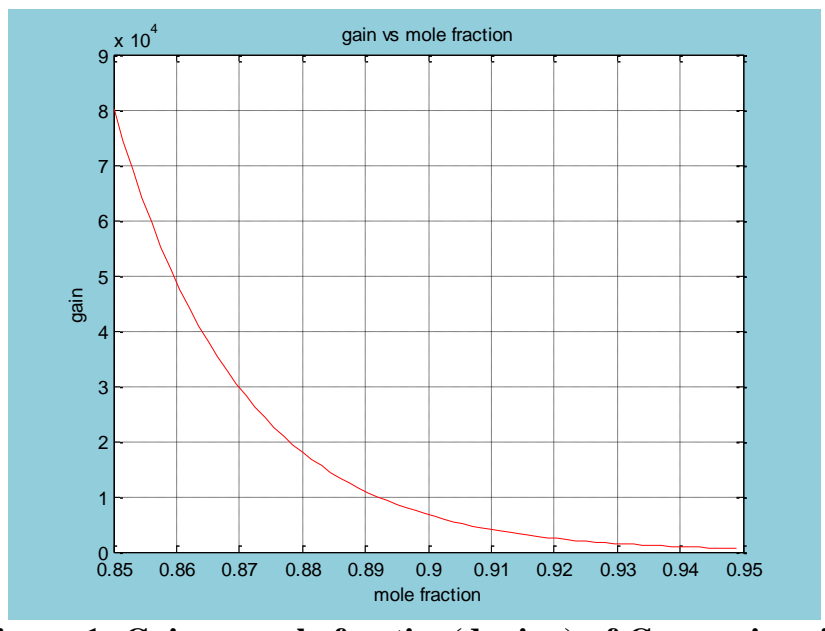

Figure 1: Gain vs mole fraction(doping) of Germanium in Silicon

Studies also made on the variation of temperature with the variation of acceptor and donor concentration with $\mathrm{Ne}<\mathrm{Nb}$ and $\mathrm{Ne}=\mathrm{Nb}$. In the figure 2 it is evident that the gain is obtainable with the variation of temperature.

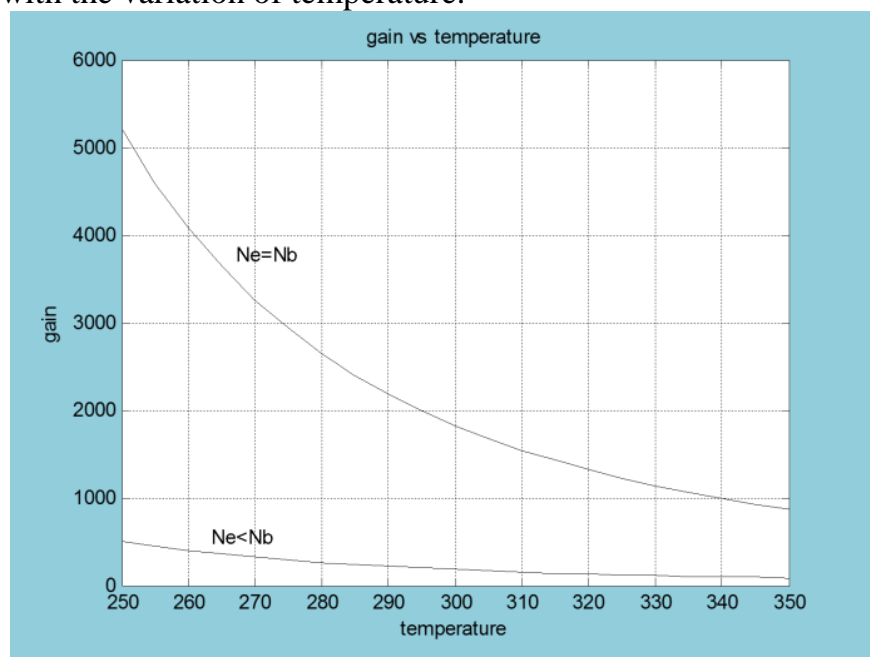

Figure 2: Gain vs Temperature for the designed heterojunction (with $\mathrm{Ne}<\mathrm{Nb}$ and $\mathrm{Ne}=\mathrm{Nb}$ )

\section{CONCLUSION}

The SiGe/Ge HBT can be used for commercial purposes because Silicon is commercially available and by the use of $\mathrm{SiGe} / \mathrm{Ge} \mathrm{HBT}$ one can achieve a better performance. This HBT is better than the existing technology. Here, lattice matching is possible, so, lattice mismatch problem can be avoided by using this HBT technology. In the current era we want features like high speed, high gain etc. By using this HBT technology we can achieve these features.

\section{ACKNOWLEDGEMENT}

We sincerely thank our ECE Department, Narula Institute of Technology for their help, support and encouragement to do this research work.

\section{REFERENCE}

[1] Shur Michael, Physics of semiconductor devices, Prentice- Hall of India Private LTD.

[2] Kroemer Herbert, Dec 08, 2000,"Quasi- Electric fields \& Band Offsets: Teaching Electrons New Tricks", Nobel Lecture, - ECE Deptt. University of California.

[3] Kroemer H., Dec. 1963, "A proposed class of heterojunction injection lasers," Roc. ZEEE,vol. 51, pp. 1782-1783.

[4] Patri V.S., Kumar M.J., August 1998, "Profile design Considerations for Minimizing Base transit time in SiGe HBTs", Transactions on Electron Devices , Vol. 45, No. 8.

[5] Lee $\mathrm{K}$ et all, 2006, "Improved VBIC Model for SiGe HBTs With a Unified Model of Heterojunction Barrier Effects", IEEE Transactions on Electron Devices, vol. 53, no.4, 743.

[6] Sze S.M., 2005, Semiconductor Devices, Physics and Technology John Wiley and Sons, Inc, , pp. 151.

[7] Aurora N.D., Houser J.R. and Roulston D.J., 1982, " Electron and Hole Mobilities in Silicon as a Function of Concentration and temperature", IEEE Transactions on Electron Devices ,vol.ED29,no.2,pp 292.

[8] Kasper E. , 1995, "Properties of Strained and Relaxed Silicon Germanium", INSPEC, London.

[9] Kroemer H., Jan. 1981,"Heterostructures for everythingdevice principle of the 1980's?," Japan J. AppL Phys., vol. 20, suppl. 20-1, pp. 9-13.

[10] Katoda T. and Kishi M., Apr. 1980, "Heteroepitaxial growth of gallium phosphide on silicon," J. Electron Mat., vol. 9 , no. 4, pp. 783-796 .

[11] Basu Sukla , Oct. 13-15, 2008,"Analytical modeling of base transit time of SiGe HBTs including effect of temperature", International Semiconductor Conference, CAS 2008, held on. Sinaria, Romania, vol. 2, pp. 339342.

[12] Cheung D. T., Chiang S. Y., and Pearson G. L., Mar. 1975 "A simplified model for graded-gap heterojunctions," Solid-State Electron., vol. 18, no. 3, pp. 263-266. 